\title{
CRITICAL ANALYSIS OF THE CLUSTER INTEGRAL APPROACH TO THE NEMATIC STATE $\left({ }^{*}\right)$
}

\author{
A. SAUPE \\ Liquid Crystal Institute, Kent State University, Kent, Ohio 44242 U.S.A.
}

\begin{abstract}
Résumé. - La théorie d'Onsager pour les sélections de particules anisotropes est basée sur la théorie de Mayer d'intégrales d'amas pour les gaz imparfaits. L'énergie libre est exprimée comme une série d'intégrales d'amas irréductibles qui peuvent être considérées comme indépendantes du volume. Notre étude montre que l'hypothèse d'indépendance n'est pas justifiée pour les solutions considérées à des concentrations proches de la transition nématique isotrope. Négliger la dépendance en volume conduit probablement en général à des erreurs qui favorisent la formation d'un état nématique. Une étude de la fonction de corrélation de paires montre qu'aucun ordre orientationnel à longue distance ne peut exister si le nombre d'intégrales d'amas irréductibles non nulles est fini. Contrairement aux résultats d'Onsager, un traitement théorique exact ne peut donc donner une phase nématique lorsque seule la première intégrale d'amas irréductible est non nulle.
\end{abstract}

\begin{abstract}
The theory by Onsager for the solution of anisometric particles is based on Mayer's cluster integral theory of imperfect gases. The free energy is expressed as a series of irreducible cluster integrals which requires that all relevant cluster integrals can be considered as volume independent. Our studies show that the assumption of volume independence is not justified for the considered solutions at concentrations close to the isotropic-nematic transition. The neglection of the volume dependence leads probably in general to errors which favor the formation of a nematic state. A study of the pair correlation function shows that no long range orientational order can exist when only a limited number of irreducible cluster integrals are different from zero. In contrast to Onsager's results an exact theoretical treatment can accordingly not give a nematic phase when only the first irreducible cluster integral is different from zero.
\end{abstract}

1. Introduction. - Onsager's theory [1] for nematic solution of anisometric particles accounts for the effect of the particle shape by treating the system similar to an imperfect gas. The theory by Flory [2] uses a different approach. It is based on a lattice model. Both theories give similar results and explain the formation of nematic states in solutions of rod-shape particles. (For a review and comparison see for instance ref. [3].)

Flory's theory can only give approximate results since it is based on a simplified model. The cluster integral expansion used by Onsager may in principle give accurate results, that is, when the expansion can be extended to sufficiently high terms and provided that in the density range of interest the cluster integral approach is still applicable. The discussion of the latter part is the main objective of this paper.

2. Volume dependence of cluster integrals. - In Onsager's theory the solution of anisometric particles

$\left(^{*}\right)$ This research was supported in part by a grant from the National Science Foundation \# DMR-76-21363. are treated like a classical gas of rigid particles. The free energy in this case can be written in the form

$$
F=-T N \log \varphi(T)-T \log \left(Q_{\tau} / N !\right) .
$$

Here $\varphi(T)$ depends only on temperature.

$$
\left(\varphi(T)=(2 \pi T)^{3}\left(m^{3} A B C\right)^{1 / 2} 8 \pi^{2} / h^{6}\right.
$$

for a gas of anisometric rigid particles, $m$ mass, $A, B, C$ principle inertial moments.) $Q_{\tau}$ stands for the configuration integral

$$
Q_{r}=\int \mathrm{e}^{-U / T} \mathrm{~d} V^{(N)} \mathrm{d} \Omega^{(N)}
$$

The integration is over volume and orientation of the particles (in terms of Euler angles

$$
\left.\mathrm{d} \Omega=\sin \theta \mathrm{d} \theta \mathrm{d} \varphi \mathrm{d} \psi / 8 \pi^{2}\right) .
$$

We assume a pairwise interaction so that $U=\sum_{i>j} u_{i j}$ where $u_{i j}$ depends only on the coordinates and orientation of particle $i$ and $j$. With this assumption $Q_{\tau}$ can be expressed in terms of cluster integrals (for detail derivations see refs. [4-6])

$$
\frac{Q_{\tau}}{N !}=\sum_{m_{l}} \prod_{l} \frac{\left(N v b_{l}\right)^{m_{l}}}{m l !}
$$


Here the sum is to be taken over all products for which $\sum l m_{l}=N$ and $v=V / N$. The cluster integrals $b$ are in turn defined by

$$
b_{l}=\frac{1}{V l !} \int U^{(l)}\left(V^{(l)}, \Omega^{(l)}\right) \mathrm{d} V^{(l)} \mathrm{d} \Omega^{(l)} .
$$

The Ursell functions $U^{(t)}$ are products of the factors $f_{i j}=\mathrm{e}^{-u_{i j} / T}-1$. It is

$$
\begin{aligned}
& U^{(1)}=1 \\
& U^{(2)}=f_{12} \\
& U^{(3)}=f_{12} f_{13}+f_{12} f_{23}+f_{13} f_{23}+f_{12} f_{13} f_{23}
\end{aligned}
$$

and in general $U^{(l)}=\sum \prod f_{i j}$ where the sum is to be taken over all products that correspond to clusters of $l$ molecules, that is over products in which the indices of all $l$ molecules occur and which cannot be divided into two factors that have no index in common.

Eq. (3) is generally valid under the assumption of pairwise interaction. For the further development of the formalism it has to be assumed that the cluster integrals (4) which are in general volume dependent can be replaced by the volume independent values which are obtained when the volume integration for all but one particle is taken over an infinitely extended volume. Under this assumption one can derive

$$
F=N T[\log Z+\log \varphi(T)]-V \sum_{l} b_{l} Z^{l} .
$$

Where the fugacity $Z$ is given by

$$
\sum \frac{l V}{N} b_{l} Z^{l}=1 \text {. }
$$

The final result in terms of irreducible cluster integrals is

$F=N T\left[\log \frac{N}{V}-\log \varphi(T)-1-\sum_{k>1} \frac{\beta_{k}}{k+1}\left(\frac{N}{V}\right)^{k}\right]$

with

$$
\beta_{k}=\frac{1}{k !} \sum \prod f_{i j} \mathrm{~d} V^{(k+1)} \mathrm{d} \Omega^{(k+1)},
$$

where the sum is over all products corresponding to irreducible clusters of $k$ molecules. Such products cannot be divided into two parts that have no index in common after removal of any one of the factors $f_{i j}$ of the original product. In the nematic state the configurations are limited to the states with long range orientational order. The cluster integrals under this constraint are given by

$$
\begin{aligned}
b_{l}=\frac{1}{V l !} \int & U^{(l)}\left(V^{(l)}, \Omega^{(l)}\right) \times \\
& \times \mathrm{d} V_{1} \ldots \mathrm{d} V_{l} w\left(\Omega_{1}\right) \mathrm{d} \Omega_{1} \ldots w\left(\Omega_{l}\right) \mathrm{d} \Omega_{l}
\end{aligned}
$$

and the free energy in terms of irreducible cluster integrals [1]

$$
\begin{aligned}
F & =N T[\log N-\log \varphi(T)-1- \\
& \left.-\int w(\Omega) \log w(\Omega) \mathrm{d} \Omega-\sum \frac{\beta_{k}}{k+1}\left(\frac{N}{V}\right)^{k}\right] .
\end{aligned}
$$

Where $w(\Omega)$ denotes the normalized orientational distribution function and the modified irreducible cluster integrals are determined by

$$
\begin{aligned}
\beta_{k}=\frac{1}{k !} \sum \int \prod f_{i j} \mathrm{~d} V_{1} \ldots \mathrm{d} V_{k+1} w\left(\Omega_{1}\right) \times \\
\times \mathrm{d} \Omega_{1} \ldots w\left(\Omega_{k+1}\right) \mathrm{d} \Omega_{k+1} .
\end{aligned}
$$

We return now to the discussion of the volume dependence of the cluster integrals (4) or (9). The functions $f_{i j}$ are zero for non-interacting particles. To evaluate a cluster integral of $l$ molecules we may integrate first over the orientations of all particles and over the positions of the first $l-1$ particles. The result of this integration is independent of the volume when the distance of particle $(l)$ from the boundary is larger than $(l-1) r^{*}$ where $r^{*}$ is the interaction distance between the particles. It results from the fact that $f_{i j}$ is zero for non-interacting particles that is for $r_{i j}>r^{*}$. The integrand of (4) is therefore always zero when the distance of any particle of the cluster from particle $(l)$ is larger than $(l-1) r^{*}$. For such positions of particle (l) no error results when the integration limits for the $(l-1)$ first particles are extended to an infinite volume. On the other hand, when the distance of $(l)$ from the boundary becomes smaller than $(l-1) r^{*}$ the result of the integration depends on the volume and is modified when the limits are extended to infinity. As the error occurs only in a boundary range, it can be made small when at fixed $l$ the volume is chosen large enough.

The order of the clusters in dilute gases that are important for the configuration integral is independent of the volume. The use of volume independent cluster integrals is then well justified. In the other limit when the density is high so that the average distance between the particles is smaller than their interaction range the clusters of importance will comprise practically all molecules. The volume dependence is then significant and the expressions for the free energy [Eq. (5), (7) and (10)] are incorrect.

We are in particular interested in cylindrical particles of a large length to diameter ratio $(L / D>50)$ which interact like hard rods. For an interaction the distance between the centers of the particles must be smaller than the length $L$. The interaction range is accordingly equal to $L$. It means for the above considered cluster integral that the integration the first $(l-1)$ particles of a cluster integral depends on the volume when the distance from the boundary of particle $l$ is smaller than $(l-1) L$. It is convenient to assign to the particles 
an interaction volume. In the given case this may be set equal to $v^{*}=\frac{\pi}{6} L^{3}$. The cluster integral approach is accordingly justified when $V / N=v \gg v^{*}$.

Onsager's theory for a system of hard rods with $L \gg D$ gives for the particle density of the isotropic phase at the transition point [3] $\rho_{\text {iso }}=4.2 / L^{2} D$ and for the nematic phase in equilibrium with the isotropic phase $\rho_{\text {nem }}=5.3 / L^{2} D$. It follows that at the transition

$$
\begin{aligned}
& v^{*} / v_{\text {iso }}=2 L / D \\
& v^{*} / v_{\text {nem }}=3 L / D .
\end{aligned}
$$

Both values are large compared to 1 and not small as required for the validity of a cluster integral expansion. It should be noticed that the volume of the particle itself is small compared to $v_{\text {iso }}$ or $v_{\text {nem }}$ $\left(v_{\text {part }} / v_{\text {iso }} \approx 3 D / L\right)$.

The above estimate for $v^{*}$ may be too stringent and it may be better to use the co-volume of two particles [1] as an estimate for an effective interaction volume. We obtain in this way

$$
\begin{aligned}
& v^{*} / v_{\text {iso }}=6 \\
& v^{*} / v_{\text {nem }}=2 .
\end{aligned}
$$

It has been assumed here that the degree of order in the nematic phase at the transition is 0.78 as given by the theory [3]. The volume ratios are independent of $L / D$ in the limit of a large length to width ratios but they are not small compared to 1 .

It does not seem possible to make a quantitative estimate of the error that results from the volume dependence of the cluster integrals. Probably the unjustified extension of the integration limits results in too large values for the excluded columns. Judging from the ratios (13) the error will be larger for the isotropic state than for the ordered state where the effective interaction volume is reduced because of the partial parallel alignment. The procedure favors accordingly the ordered state. It will consequently predict phase transition at too low densities and give theoretical $S$-values that are too high. The conclusion that the theories favor the ordered state is also supported by a study of the pair correlation function.

3. Pair correlation and irreducible cluster integrals. - We consider the correlation between particle (1) and (2). For this purpose we write the interaction potential in the form

$$
U=u_{12}+\sum_{i>j>3} u_{i j}+\sum_{k \geqslant 3} \sum_{k=1,2} u_{k} .
$$

The pair correlation function can be expressed in terms of products in a similar way as the configuration integral (see for instance ref. [6] chapter X).

$$
\begin{aligned}
g^{(2)} & =\frac{V^{2}}{Q_{\tau}} \int \mathrm{e}^{-U / T} \mathrm{~d} V_{3} \ldots \mathrm{d} V_{N} \mathrm{~d} \Omega_{3} \ldots \mathrm{d}_{N} \\
& =\frac{V^{2}}{Q_{\tau}} \mathrm{e}^{-u_{12} / T} \int \sum \prod f_{i j} f_{k \kappa} \mathrm{d} V_{3} \ldots \mathrm{d} V_{N} \mathrm{~d} \Omega_{3} \ldots \mathrm{d} \Omega_{N}
\end{aligned}
$$

The index $\kappa$ in this expression can assume the values 1 and 2 while $i, j, k$ may assume all values $\geqslant 3$. The products $\prod f_{i j} f_{k \kappa}$ define again a division of the system into clusters. Only the clusters that contain particle (1) and (2) contribute to the correlation between the particles.

We consider now the orientational correlation between the two particles when they are in a fixed position a distance $r=m r^{*}$ appart. Here $r^{*}$ is again the interaction range. A cluster that contributes to the correlation.must then contain $l \geqslant m-1$ particles in addition to the two particles that it connects. The integrals over clusters containing the two particles

$$
\begin{aligned}
& c_{l, 2}\left(r_{1} \Omega_{1}, r_{2} \Omega_{2}\right)=\frac{1}{\left(8 \pi^{2}\right)^{l}} \times \\
& \quad \times \int \prod f_{i j} f_{k \kappa} \mathrm{d} V_{3} \ldots \mathrm{d} V_{l+2} \mathrm{~d} \Omega_{3} \ldots \mathrm{d} \Omega_{l+2}
\end{aligned}
$$

cannot all disappear when correlation exists as we assume here. We select a cluster that makes a finite contribution and choose a second set of particles with which we form a product that corresponds to an equivalent cluster containing particle (1) and (2) (see Fig. 1). Multiplying this product with original

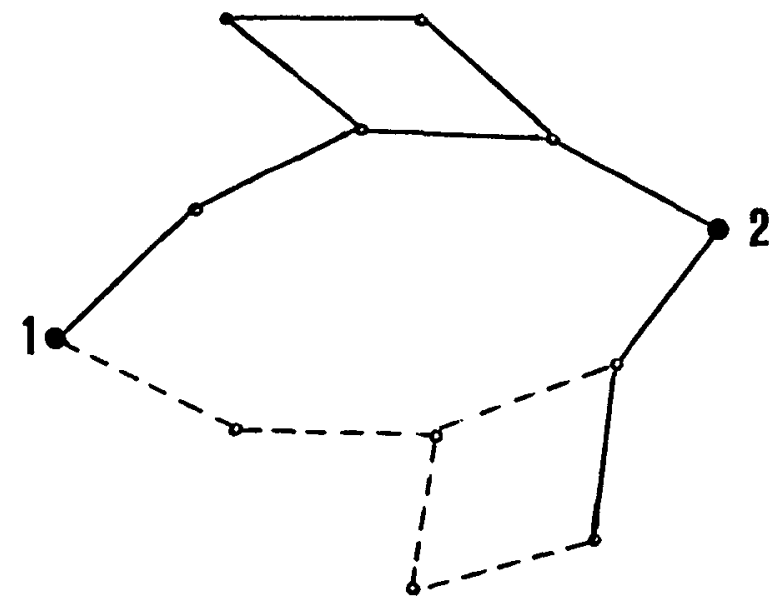

FIG. 1. - Graphical representation of a cluster connecting particle (1) and (2), and complementary cluster of a second set of particles.

product we obtain a cluster of $2 l$ particles in addition to (1) and (2). We integrate now over the $2 l$ particles holding the position of the first two particles fixed. This gives

$$
\begin{array}{rlrl}
c_{2 l, 2}\left(r_{1} \Omega_{1}, r_{2} \Omega_{2}\right) & =\frac{1}{\left(8 \pi^{2}\right)^{2 l}} \int \prod f_{i j} f_{k \kappa} \mathrm{d} V_{3} \ldots \mathrm{d} V \Omega_{+2} \mathrm{~d} \Omega_{3} \ldots \mathrm{d} \Omega_{l+2} \times \\
& =c_{l, 2}^{2}\left(r_{1} \Omega_{1}, r_{2} \Omega_{2}\right) . & \times \int \prod f_{i^{\prime} j^{\prime}} f_{k^{\prime} \kappa} \mathrm{d} V_{l+3} \ldots \mathrm{d} V_{2 l+2} \mathrm{~d} \Omega_{l+3} \ldots \mathrm{d} \Omega_{2 l+2}
\end{array}
$$


Finally, we integrate $c_{2 l, 2}$ over the positions and orientations of particle (1) and (2). The integral corresponds to one of the terms of $b_{2 l+2}$, the cluster integral of the first kind which we considered in the previous section. It has a positive value since according to our assumption $c_{t, 2}$ does not identically disappear. The value of such an integral is equal to a product of integrals over irreducible clusters. In our case, one of these irreducible clusters must be of order $k>2 m-1$ or larger because the cluster contains a loop of $\mathrm{k}+1>2 \mathrm{~m}$ bonded particles (see Fig. 1).

Our considerations show that a long range orientational order cannot exist when the irreducible cluster integral above a certain order disappears. It contradicts Onsager's result which predicts a nematic phase using only the first irreducible cluster integral. The contradiction probably results from the use of the cluster integral approach at too high densities.
4. Conclusion. - It has been shown that the cluster integral expansion does not provide a sound basis for a theory of nematic states because the particle density is too high in the range where the transition occurs. This is true also for system of long cylindrical particles. In spite of this difficulty the method gives results which are in reasonable agreement with the observations. For instance, it explains successfully the correlation between particle density at the transition point and length to width ratio of the particles. On the other hand, the predicted degree of order for the nematic state seems much too high. The partial success shows that the cluster expansion can be useful beyond its range of accurate validity but a theoretical justification is still lacking.

\section{References}

[1] Onsager, L., Ann. N.Y. Acad. Sci. 51 (1949) 627.

[2] Flory, P. J., Proc. R. Soc. (London) A 234 (1956) 73.

[3] Srraley, J. P., Mol. Cryst. Liq. Cryst. 22 (1973) 333.

[4] MAYer, J. E. and Goepert-Mayer, M., Statistical Mechanics, New York, 1948.
[5] MAYER, J. E., Theory of Real Gases in Encyclopedia of Physics, Ed. Flügge, S. (Springer-Verlag, Berlin) 1958, vol. 12. [6] MünSter, A., Statistical Thermodynamics (Springer-Verlag, Berlin) 1969, Vol. 1, Chapter IX. 